\title{
MATTER IN THE BEAM: WEAK LENSING, SUBSTRUCTURES, AND THE TEMPERATURE OF DARK MATTER
}

\author{
Hareth S. Mahdi ${ }^{1,2}$, Pascal J. Elahi ${ }^{1}$, Geraint F. Lewis ${ }^{1}$, and Chris Power $^{3}$ \\ ${ }^{1}$ Sydney Institute for Astronomy, School of Physics, A28, The University of Sydney, NSW 2006, Australia; hareth@physics.usyd.edu.au \\ ${ }^{2}$ Department of Astronomy, University of Baghdad, Jadiryah, Baghdad 10071, Iraq \\ ${ }^{3}$ International Centre for Radio Astronomy Research, University of Western Australia, 35 Stirling Highway, Crawley, WA 6009, Australia \\ Received 2015 December 3; revised 2016 April 15; accepted 2016 May 24; published 2016 August 1
}

\begin{abstract}
Warm dark matter (WDM) models offer an attractive alternative to the current cold dark matter (CDM) cosmological model. We present a novel method to differentiate between WDM and CDM cosmologies, namely, using weak lensing; this provides a unique probe as it is sensitive to all of the "matter in the beam," not just dark matter haloes and the galaxies that reside in them, but also the diffuse material between haloes. We compare the weak lensing maps of CDM clusters to those in a WDM model corresponding to a thermally produced $0.5 \mathrm{keV}$ dark matter particle. Our analysis clearly shows that the weak lensing magnification, convergence, and shear distributions can be used to distinguish between CDM and WDM models. WDM models increase the probability of weak magnifications, with the differences being significant to $\gtrsim 5 \sigma$, while leaving no significant imprint on the shear distribution. WDM clusters analyzed in this work are more homogeneous than CDM ones, and the fractional decrease in the amount of material in haloes is proportional to the average increase in the magnification. This difference arises from matter that would be bound in compact haloes in CDM being smoothly distributed over much larger volumes at lower densities in WDM. Moreover, the signature does not solely lie in the probability distribution function but in the full spatial distribution of the convergence field.
\end{abstract}

Key words: cosmology: theory - dark matter - galaxies: clusters: general - gravitational lensing: weak - methods: numerical

\section{INTRODUCTION}

Tensions between observations and predictions from the cold dark matter (CDM) cosmological model has renewed interest in other types of dark matter (e.g., Finkbeiner et al. 2011; Baldi 2012; Lovell et al. 2012; Vogelsberger et al. 2012; Libeskind et al. 2013; Macciò et al. 2013; Vogelsberger \& Zavala 2013; Carlesi et al. 2014a, 2014b). One particular flavor of great interest is warm dark matter (WDM), where the fundamental DM particle has an appreciable velocity at early times. One of the best-known examples is a sterile neutrino, which could explain observed neutrino oscillation rates and baryogenesis ( $\nu$ MS; e.g., Asaka et al. 2006). WDM models are not only attractive due to their ability to address issues in particle physics, but also for their effect on cosmological structure formation. The non-negligible motions leave a specific feature in the initial density perturbations from which dark matter haloes arise, namely, a suppression of power in the density field below the so-called free-streaming scale.

As a consequence, WDM models predict negligible numbers of haloes below the free-streaming scale (Boylan-Kolchin et al. 2012; Schneider et al. 2012; Libeskind et al. 2013; Lovell et al. 2014). This suppression may reconcile the difference between the observed and predicted number of low-mass dwarf galaxies around large galaxies such as our own Milky Way, solving the so-called missing satellites problem (e.g., Klypin et al. 1999; Moore et al. 1999). For instance, Lovell et al. (2012) showed that the resonantly produced sterile neutrino WDM models, with particle masses of $\sim 2 \mathrm{keV}$ compatible with the Ly $\alpha$ bounds (Boyarsky et al. 2009a, 2009b), decrease the number of substructures residing in a MW-size halo, significantly alleviating the missing satellite problem. Although the simple suppression of power cannot alone account for all of the small-scale discrepancies (e.g., Schneider et al. 2014), this family of models has the advantage of possibly explaining several other observational anomalies, such as the $3.5 \mathrm{keV}$ X-ray line seen in clusters (e.g., Bulbul et al. 2014).

Although most studies have focused on the effect of WDM on small-scale cosmic structures, the absence of small-scale power leaves its fingerprints on scales much larger than the free-streaming scale (e.g., Obreschkow et al. 2013). Signatures of WDM at larger scales have generally been neglected since one of the desired features of WDM models is that they have the same large-scale matter distribution as CDM models while possibly resolving discrepancies on small scales.

Gravitational lensing is one such probe as it is sensitive to the entire underlying matter distribution (e.g., Bartelmann \& Schneider 2001; Schneider 2003; Ellis 2010). In Mahdi et al. (2014) and Elahi et al. (2014), we showed that strong lensing by galaxy clusters can differentiate between WDM and CDM models. WDM clusters have larger Einstein radii and lensing cross-sections. This result is contrary to the naive expectation that WDM clusters should have smaller strong lensing crosssections than their CDM counterparts as CDM clusters contain more subhaloes, and subhaloes increase the lensing crosssection (Xu et al. 2009). This unexpected signature reduces the tension between observations and theory: observed clusters produce more lensed giant arcs than predicted by $\Lambda \mathrm{CDM}$, that is, the so-called arc statistics problem (Bartelmann et al. 1998; see Meneghetti et al. 2013 for a review). WDM decreases this tension, although it does not fully resolve it.

In this paper, we continue exploring novel approaches for probing signatures of WDM, specifically one that does not rely on rare alignments between galaxies required for strong lensing, namely, weak lensing. Weak lensing is a powerful tool for probing our cosmology as it is sensitive not just to the density peaks corresponding to haloes but to all of the matter in 
the beam. Historically, it has been measured using the shear of background galaxies through the statistical correlation of observed galaxy ellipticities (e.g., Sheldon et al. 2009; Umetsu et al. 2014; and see Bartelmann \& Schneider 2001 for a review). The small weak lensing magnification has primarily been detected through its effect on the number density of a flux-limited sample (e.g., Hildebrandt et al. 2009), although more recent observations have made use of the effect on the observed magnitudes and sizes of background galaxies around lensing clusters (e.g., Schmidt et al. 2012), and the distortion in the shape of background galaxies (e.g., Heymans et al. 2012; Gruen et al. 2014). Gillis \& Taylor (2016) showed that the weak magnification signal can be estimated by comparing the number density of galaxies in a patch of sky with the expected unlensed number density.

Several techniques of cosmic shear measurements have been investigated by the Shear TEsting Program (STEP; Heymans et al. 2006; Massey et al. 2007) and GRavitational lEnsing Accuracy Testing (GREAT; Bridle et al. 2010). These studies highlight observational problems such as blurring, pixelization, and noise uncertainty, which must be taken into account in order to measure the shear with high accuracy. The problems are somewhat alleviated by stacking images, which increases the signal-to-noise ratio (Lewis 2009).

Furthermore, due to the small distortion caused by weak lensing, detecting the weak lensing signal with high signal to noise requires a large number of background galaxies. The Dark Energy Survey (DES) aims to reconstruct the cosmic shear using approximately 300 million galaxies by surveying an area 30 times larger than previous weak lensing surveys (Flaugher 2005). This will eventually be supplanted by LSST, which will use billions of galaxies (LSST Science Collaboration et al. 2009).

Here, we demonstrate how the weak lensing magnification distribution provides a novel probe to measure the "temperature" of dark matter. First, we present our methods in Section 2, and then our results in Section 3. We conclude in Section 4.

\section{SYNTHETIC WEAK LENSING MEASUREMENTS}

We study the weak lensing signature around galaxy clusters from a suite of cosmological zoom simulations using a ray tracing method. We stack the weak lensing from our clusters along multiple lines of sight and compare the magnifications in our two models.

\subsection{Simulations}

We use 10 pairs of clusters extracted from zoom simulations of $\Lambda \mathrm{WDM}$ and $\Lambda \mathrm{CDM}$ cosmologies. Here, we briefly discuss our simulations; for further details see Elahi et al. (2014). The cosmological parameters used were $h=0.7, \Omega_{m}=0.3$, $\Omega_{\Lambda}=0.7$, and $\sigma_{8}=0.9$. The WDM model used is a $0.5 \mathrm{keV}$ thermally produced dark matter particle (Bode et al. 2001; Power 2013) which results in a suppression of the growth for a halo with $M \lesssim M_{\mathrm{hm}}=2.1 \times 10^{11} M_{\odot}$, i.e., the so-called halfmode mass scale where the WDM power spectrum is $1 / 4$ that of the CDM one (Schneider et al. 2012). Based on phase-space considerations, Shao et al. (2013) estimated the mass of the WDM particle to be $\sim 0.5 \mathrm{keV}$. WDM cosmologies with a dark matter mass of $0.5 \mathrm{keV}$ have been explored by various studies (e.g., Schneider et al. 2012; Viel et al. 2012; Kang et al. 2013).
We note that the WDM initial conditions do not include nongravitational velocities, and thus, technically, the WDM simulations are CDM simulations with a smooth truncation in the initial density perturbation power spectrum at a scale corresponding to $0.75 h^{-1} \mathrm{Mpc}$. All of the simulations were run with GADGET2, a TreePM code (Springel 2005), and each pair of zoom simulations used the same gravitational softening length based on Power et al. (2003), i.e., $\epsilon_{\mathrm{opt}}=4 R_{\mathrm{vir}} / \sqrt{N_{\mathrm{vir}}}$, using $R_{\text {vir }}$ from the parent CDM simulation. Clusters and their subhaloes are identified using VELOCIRAPTOR (also known as STF; Elahi et al. 2011).

\subsection{Weak Lensing}

Gravitational lensing probes the surface mass density of the lens $\Sigma$, specifically through the lensing potential $\psi$ along a line of sight (los):

$$
\psi(\boldsymbol{\theta})=\frac{1}{\pi} \int \kappa\left(\boldsymbol{\theta}^{\prime}\right) \ln \left|\boldsymbol{\theta}-\boldsymbol{\theta}^{\prime}\right| d^{2} \theta^{\prime},
$$

where $\kappa=\Sigma / \Sigma_{\text {crit }}$ is the convergence, $\Sigma_{\text {crit }}=\frac{c^{2}}{4 \pi G} \frac{D_{\mathrm{S}}}{D_{\mathrm{L}} D_{\mathrm{LS}}}$ is the critical surface mass density which depends on the angular distances to the source and lens, $D_{\mathrm{L}}, D_{\mathrm{S}}$ and between the lens and source $D_{\mathrm{LS}}$, and $\theta$ is the angle from the center of the lens to the los. The magnification matrix $\mathcal{A}$ of an image can be written in terms of the convergence, and the two components of shear $\gamma_{1}$ and $\gamma_{2}$ as follows:

$$
\mathcal{A}=\left(\begin{array}{ll}
1-\frac{\partial^{2} \psi}{\partial \theta_{x}^{2}} & \frac{\partial^{2} \psi}{\partial \theta_{x} \partial \theta_{y}} \\
\frac{\partial^{2} \psi}{\partial \theta_{x} \partial \theta_{y}} & 1-\frac{\partial^{2} \psi}{\partial \theta_{y}^{2}}
\end{array}\right)=\left(\begin{array}{ll}
1-\kappa-\gamma_{1}-\gamma_{2} \\
-\gamma_{2} & 1-\kappa+\gamma_{1}
\end{array}\right) .
$$

The convergence gives the isotropic (de)magnification of an image due to the contribution of mass inside a bundle of light from a background source and the shear is responsible for the anisotropic distortion of images due to contribution of matter outside the bundle of light.

The total magnification of an image is given by $\mu=1 / \operatorname{det}|\mathcal{A}|=\left[(1-\kappa)^{2}-\gamma^{2}\right]^{-1}$, where $\gamma=\gamma_{1}+i \gamma_{2}$ is the total shear. Note that for a simple azithumally symmetric lens, the equations simplify and one can show that $\gamma=[\bar{\Sigma}(<r)-\Sigma(r)] / \Sigma_{\mathrm{cr}}$, that is, the shear probes the difference of the surface mass density. The weak lensing regime of a galaxy cluster takes place at a large distance from the center where $(\kappa \ll 1$ and $\gamma \ll 1)$.

The weak lensing shear has a direct impact on the observed ellipticity of background sources. The observed ellipticity of a source $\left(e_{\mathrm{obs}}\right)$ is a combination of the source galaxy's intrinsic ellipticity $\left(e_{s}\right)$ and the effect of shear,

$$
e_{\mathrm{obs}}=\frac{e_{s}+g}{1+g^{*} e_{s}},
$$

where $g$ is the reduced shear, $g=\gamma /(1-\kappa)$, and $g^{*}$ is its complex conjugate.

We briefly outline our method for constructing weak lensing maps here; for a detailed description, see Mahdi et al. (2014). We place our lensing clusters at $z_{\mathrm{L}}=0.3$ and project all of the particles within a radius of $4 \mathrm{Mpc}$ of the cluster center onto a two-dimensional grid for 48 los to overcome the magnification bias. The projected density field is smoothed by a truncated 


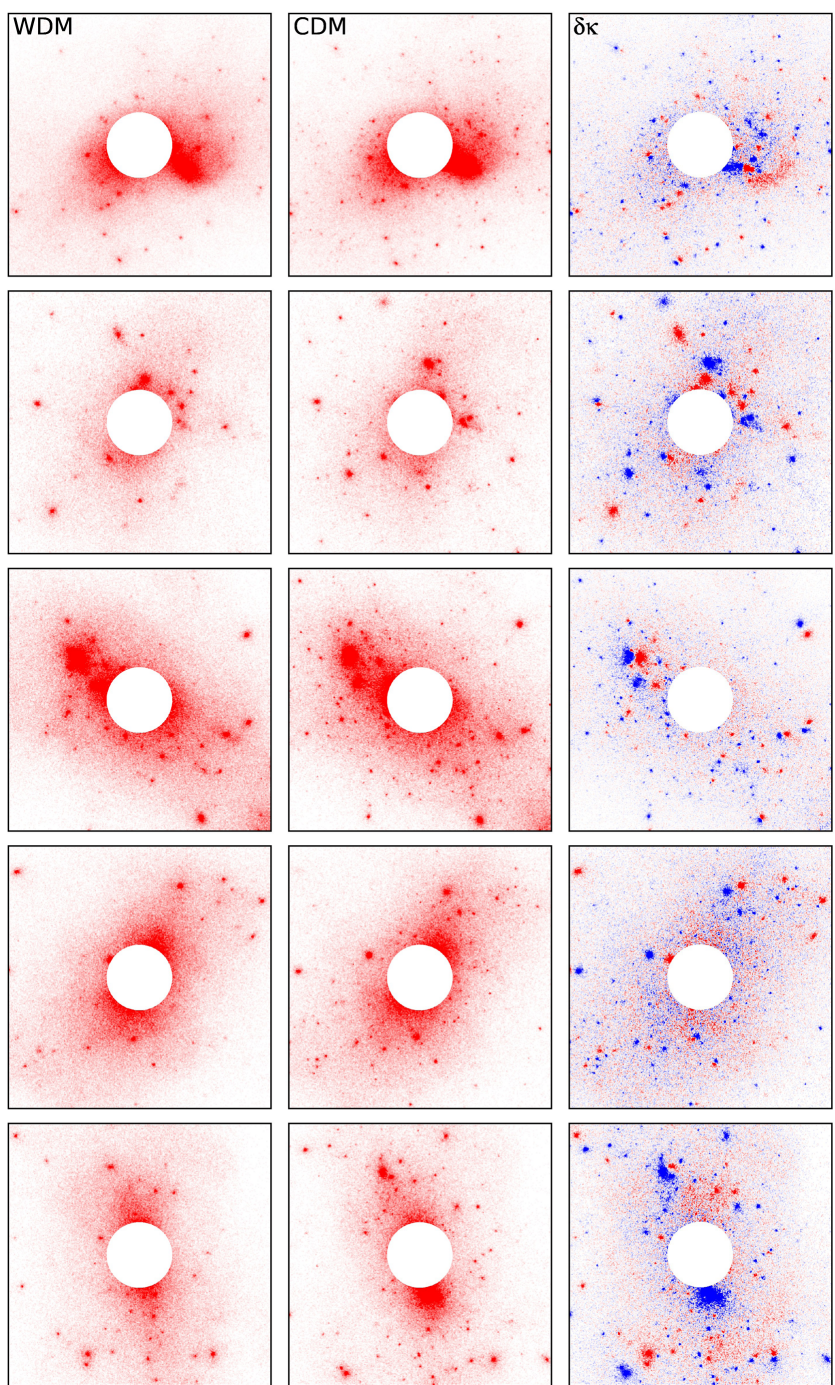

Figure 1. Convergence maps of five WDM clusters (left column) and their CDM counterparts (middle column) as well as the difference between WDM and CDM, $\delta \kappa \equiv \kappa_{\mathrm{WDM}}-\kappa_{\mathrm{CDM}}$ (right column). The side length of each map is $4 \mathrm{Mpc}$ and the strong lensing regions (i.e., within $1 \mathrm{Mpc}$ in diameter) are excluded. Here, pixels with $\delta \kappa>0(<0)$ are red (blue).

Gaussian kernel with a smoothing scale of $5 h^{-1} \mathrm{kpc}$ in order to overcome the numerical noise due to the discreteness of $\mathrm{N}$ body simulation. We use a $8192^{2}$ grid, resulting in an angular resolution of 0.22 arcsec per pixel.

The convergence produced by five pairs of clusters along a single los as well as the difference $\delta \kappa \equiv \kappa_{\mathrm{WDM}}-\kappa_{\mathrm{CDM}}$ is shown in Figure 1. To emphasize the differences in the weak lensing regime, we mask out the strong lensing region (1 Mpc in diameter). We see that in both cosmologies, the clusters contain small regions of high convergence, corresponding to the large subhaloes. As both the WDM and CDM simulations used the same phases in the initial conditions, every large subhalo in a CDM cluster has a counterpart in the WDM analog. This is why large, compact red regions $(\delta \kappa>0)$ are paired with a blue region $(\delta \kappa<0)$ in the difference map. The key feature is that more pixels with $\delta \kappa>0$ are smoothly distributed about the clusters. We find that $53 \%$ of the surface area has $\delta \kappa>0$ for all clusters in our sample. We will discuss this feature in the following sections.
For each projection, we distribute 30,000 elliptical sources (galaxies) of random orientation and ellipticity on the source plane at $z_{\mathrm{S}}=2$ (i.e., 30 galaxies per square arcmin, which is the effective number density practicable for weak lensing surveys; see, e.g., LSST Science Collaboration et al. 2009). We use the lens equation $\boldsymbol{\beta}=\boldsymbol{\theta}-\boldsymbol{\alpha}(\boldsymbol{\theta})$ to trace light rays of every single source from the source plane to the lens plane, identify the corresponding image(s) by means of a componentlabeling algorithm that was proposed by Chang et al. (2004). The convergence and shear of an identified image are calculated by averaging over all of the pixels that correspond to the image and the magnification is calculated from $\mu=1 /\left|(1-\kappa)^{2}-\gamma^{2}\right|$.

\section{RESULTS AND ANALYSIS}

We characterize the images of a multitude of sources produced by gravitational lenses, like those in Figure 1. Naturally, outside the cluster center there is little mass above the normal background density along a given los and background galaxies are not strongly magnified. We focus on the areas where the magnification is non-negligible and we have ignored those areas where $1-\mu \lesssim 10^{-3}$. We note that the form of the probability distribution function (PDF) is the same for all of the clusters.

The very outskirts of our clusters typically contain underdense regions. These void-like regions are poorly sampled even in our simulations, resulting in areas with fewer than one particle per pixel. The convergence in these areas cannot be accurately measured and our method typically returns convergences of $\lesssim 10^{-5}$. As WDM haloes are more extended (Elahi et al. 2014) and the voids in WDM cosmologies are not as underdense as those in CDM cosmologies (Yang et al. 2015), the number of pixels with very low magnifications in our CDM simulations is artificially high. However, this does not affect our results.

To quantify the feature present in the convergence difference map seen in Figure 1, we look at the differences in the distribution of the convergence, magnification, and shear of the identified images in the weak lensing regime (i.e., images with $\kappa, \gamma \leqslant 0.05)$. We calculate the distribution of each quantity for each cluster separately and estimate the variation in the PDFs by bootstrap re-sampling using 100 sub-samples containing 1/ 5 of the total number of the identified images (Press et al. 2007). Figure 2 shows the significance in the difference between the PDFs. ${ }^{4}$ This figure shows that the convergence and magnification PDFs of WDM have an excess relative to CDM significant to $\gtrsim 5 \sigma$ for almost all clusters up to $\kappa \sim 0.01$ and $\log \mu \sim 0.01$. The one outlier is cluster 9 , which shows a much larger excess in the WDM cosmology for large magnifications. ${ }^{5}$

The difference in the shear distribution shows no significant features and the WDM and CDM PDFs are within $\lesssim 2 \sigma$. Again, cluster 9 appears to be an outlier from the rest of the clusters.

\footnotetext{
4 The first bin in this figure is affected by poorly sampled underdense regions where the convergence cannot be accurately measured, and hence the difference in it is not meaningful. Here, it happens that there are more pixels with low convergence which are poorly measured in the CDM cosmology than that in the WDM one.

5 This cluster does not appear to be very disturbed or to have a particularly unusual accretion history. However, there is a large group mass halo at the edge of the region used to calculate the weak lensing, which is closer to the main cluster in the WDM simulation. Additionally, the filamentary material bridging these objects is denser in the WDM simulation.
} 

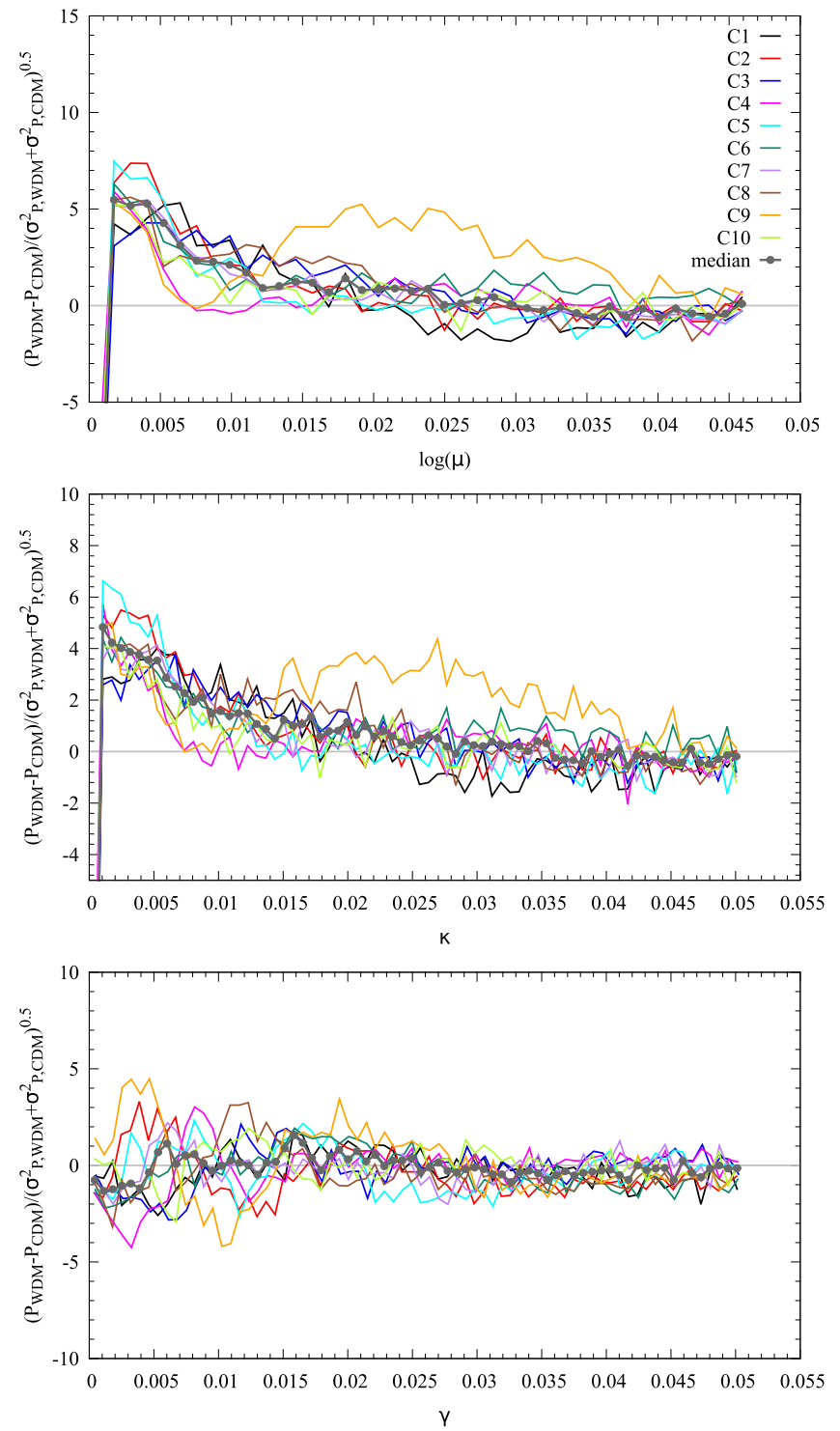

Figure 2. Significance in the difference between WDM and CDM PDFs of magnification (top), convergence (middle), and shear (bottom) for each cluster. The gray line represents the median distribution.

Where does this excess in the probability of weak lensing magnifications in WDM arise from? Figure 1 appeared to show a larger number of pixels with a positive $\delta \kappa$ between WDM and $\mathrm{CDM}$, and Figure 2 also shows that the excess magnification arises solely from a higher convergence. As $\kappa$ is sensitive to the surface density and $\gamma$ probes the changes in the surface density, the difference in the models is not due to differences in the density profiles of subhaloes or necessarily the subhaloes themselves. This higher convergence is quite simply due to there being more "matter in the beam" in regions giving rise to weak lensing. On the other hand, the fewer number of subhaloes in the WDM simulation results in fewer pixels with high shear when considering the whole shear field. However, as we focus on images in the weak lensing regime (i.e., with $\kappa$, $\gamma \leqslant 0.05)$, the shear distribution shown in the lower panel of Figure 2 is not affected.

The matter is distributed differently in WDM than it is in CDM, specifically, the material that would be bound up in compact, low-mass subhaloes is smoothly distributed over much larger volumes at lower densities. Put simply, WDM cosmologies are more homogeneous than CDM ones.

One of the first discussions of how differences in the overall matter distribution affect observations was presented by Dyer \& Roeder (1972, 1973), but this subject has been considered in a number of subsequent publications (e.g., Weinberg 1976; Nottale 1982a, 1982b, 1983; Watanabe \& Tomita 1990; Kantowski et al. 1995; Kantowski 1998; Tomita et al. 1999; Kantowski \& Thomas 2001; Brouzakis et al. 2008; Grenon \& Lake 2010; Clarkson et al. 2012; Meures \& Bruni 2012; Fleury et al. 2013). The focus of these studies was to understand how the angular diameter distance depends on the inhomogeneity of the universe, specifically on the fraction of material bound up in point masses along a los in comparison to a homogeneous background. The key finding from these studies is that inhomogeneities in the beam can provide additional (de) focusing, and hence distorting the observed relationship between redshift and the cosmological distance measures. Such inhomogeneities can have a significant influence and can even mimic the effects of dark energy on luminosity distances (for a review see Bolejko et al. 2011).

A similar effect is at play here. Consider a simple toy model for a cluster in WDM and CDM: in the WDM model, a volume contains a single large overdensity (halo), plus several less massive subhaloes randomly distributed within the volume and some smooth background; in the CDM model, the volume contains the same large halo along with a greater number of subhaloes randomly distributed within the volume. Here, we treat haloes as truncated isothermal spheres, with the halo and subhaloes having truncation radii of $0.125 L_{\mathrm{box}}$ and $0.0125 \mathrm{~L}_{\mathrm{box}}$, respectively, where $L_{\text {box }}$ is the size of the volume. For simplicity, we assume that subhaloes have the same mass. We place down $N_{\text {sub,cdm }}$ and $N_{\text {sub,wdm }}$ subhaloes in the CDM and WDM volumes and set the total mass in subhaloes to be $M_{\text {sub,tot,cdm }}=f_{\text {sub,cdm }} M_{\text {tot }}, M_{\text {sub,tot,wdm }}=f_{\text {sub,wdm }} M_{\text {tot }}$, some fraction of the total mass in the CDM and WDM, respectively. As the mass within the volume is fixed and fewer subhaloes are present in the WDM, the background density in the CDM analog is lower than that in the WDM analog by

$$
\begin{aligned}
\delta \rho_{\text {bg }} & =\left(M_{\text {sub,tot,cdm }}-M_{\text {sub,tot,wdm }}\right) / L_{\text {box }}^{3} \\
& =\left(f_{\text {sub,cdm }}-f_{\text {sub,wdm }}\right) M_{\text {tot }} / L_{\text {box }}^{3} .
\end{aligned}
$$

The change in the background density manifests as a change in the convergence. This is seen in the $\delta \kappa$ panel of the toy model where most of the volume has $\delta \kappa>0$, although the regions where $\delta \kappa<0$ have large absolute changes in the convergence.

We illustrate these effects in Figure 3 where we plot the convergence and shear of the two toy models in the two upper rows and the difference in the bottom row. We have set $N_{\text {sub,cdm }}=125$ and $f_{\text {sub,cdm }}=0.125$ in the CDM analog and $N_{\text {sub,wdm }}=25$ and $f_{\text {sub,wdm }}=0.025$ in the WDM analog. Despite the fact that there are many more overdensities in the CDM volume, most of the area has a higher convergence in the WDM toy model $(\delta \kappa>0)$. However, it is difficult to see if any bias is present in $\delta \gamma$. For this particular set of parameters, we find that $89 \%$ of the surface area has $\delta \kappa>0$ compared to $6 \%$ with $\delta \kappa<0$. The shear map is more evenly split, $42 \%$ and $58 \%$ with $\delta \gamma>0$ and $\delta \gamma<0$, respectively. Naturally, the exact numbers depend not only on $f_{\text {sub }}$ and $N_{\text {sub }}$ but the size and distribution of subhaloes. Nevertheless, this model clearly shows that the higher fraction of material bound up in compact, 

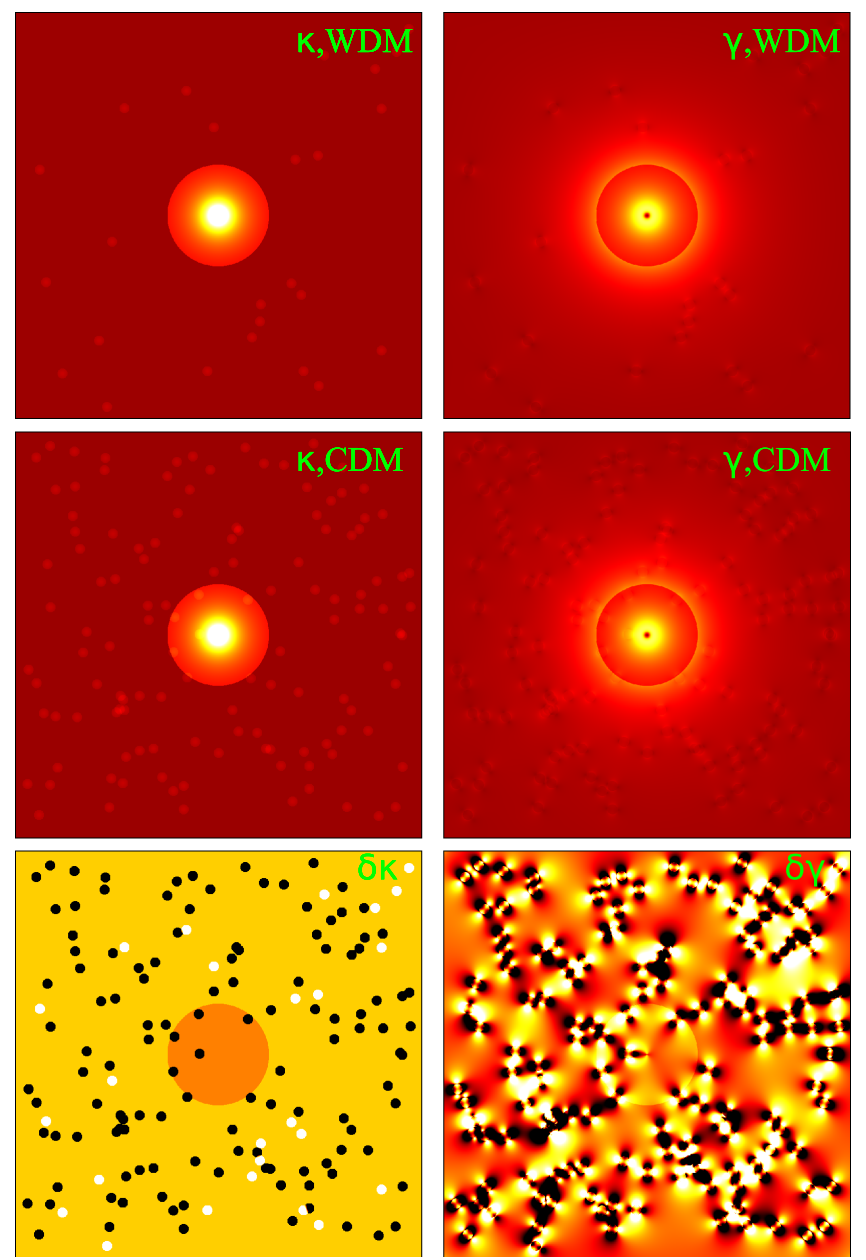

Figure 3. Lensing toy model of WDM and CDM cosmologies. The left and right columns show $\kappa$ and $\gamma$, respectively. Top: WDM analog containing a single large halo and several smaller subhaloes. Middle: CDM analog which has more small subhaloes. Bottom: differences in $\kappa$ and $\gamma$. The color scale in the two top rows are red $\sim 0$ brightening to yellow for $\gg 0$. Difference maps colors are yellow and white $=\delta \kappa, \delta \gamma>0$, orange $=\delta \kappa, \delta \gamma=0$, and black $=\delta \kappa, \delta \gamma<0$.

high-density regions in CDM cosmologies will produce smaller areas with non-negligible magnification compared to WDM cosmology. WDM leaves the shear unchanged but increases the convergence.

\section{DISCUSSION AND CONCLUSIONS}

The removal of small-scale power in WDM models has far reaching effects. It is well known that it gives rise to fewer lowmass dark matter haloes. However, the messy business of galaxy formation and evolution and the difficulty in finding small galaxies means that the galaxy luminosity function is a less than ideal probe for WDM. In this paper, we show that weak lensing offers another test for the absence of small-scale power. WDM models increase the average convergence (and magnification) in the weak lensing regime or, more specifically, the PDF distribution is shifted toward larger $\kappa$ for $\kappa \lesssim 0.01$. Moreover, the signal is not only in the PDF from the entire lensing field. The key is the fact that the spatial distributions of the convergences differ in the WDM and CDM cosmologies. It is visually apparent from the convergence maps (Figure 1) that the matter in the WDM cosmology is more homogeneously distributed than that in the CDM one.

The WDM model studied in this work assumes that the mass of WDM particles is $0.5 \mathrm{keV}$. However, several other probes prefer higher values of WDM mass particles. Nevertheless, the key finding in this paper is that the matter is distributed differently in WDM models and in turn results in a difference in the weak lensing signature. Based on the results presented in this work, one would expect the difference between the PDFs of the convergence and magnification to be smaller for WDM models with higher masses of WDM particles. A further work will consider studying the lensing characteristics of simulated clusters in WDM cosmologies with higher masses of WDM particles. This study will provide accurate statistical estimates of the difference between the CDM model and WDM cosmologies with different masses of WDM particles.

The component-labeling algorithm used in this work exactly determines how the source has been distorted by the lens. The real world, however, is not so simple as we do not know the exact shape and intensity of the source, and so these lensing quantities are hidden. Real weak lensing observations probe the reduced shear by examining the shape of the flux distribution of a galaxy (e.g., Sheldon et al. 2009; Umetsu et al. 2014) or the magnification through the number density effect (e.g., Hildebrandt et al. 2009; Ishigaki et al. 2015). A future study will explore the detectability of WDM using mock weak lensing observations of galaxy shapes and the number density of galaxies for our sample of simulated clusters. Comparing these synthetic observations with measurements from ongoing surveys such as DES and LSST would maximize the scientific impact of these measurements.

In conclusion, this study highlights the power of weak lensing as a precise cosmological probe. Weak lensing not only probes haloes but can also measure the clumpiness of the intercluster medium. Therefore, in principle, it can be used to differentiate between CDM and WDM.

H.S.M. is supported by the University of Sydney International Scholarship. P.J.E. is supported by the SSimPL programme and the Sydney Institute for Astronomy (SIfA), DP130100117 and DP140100198. C.P. is supported by DP130100117, DP140100198, and FT130100041. G.F.L. acknowledges financial support through DP130100117. This research was undertaken with the assistance of resources from the National Computational Infrastructure (NCI), which is supported by the Australian Government.

\section{REFERENCES}

Asaka, T., Shaposhnikov, M., \& Kusenko, A. 2006, PhLB, 638, 401

Baldi, M. 2012, MNRAS, 420, 430

Bartelmann, M., Huss, A., Colberg, J. M., Jenkins, A., \& Pearce, F. R. 1998, A\&A, 330, 1

Bartelmann, M., \& Schneider, P. 2001, PhR, 340, 291

Bode, P., Ostriker, J. P., \& Turok, N. 2001, ApJ, 556, 93

Bolejko, K., Célérier, M.-N., \& Krasiński, A. 2011, CQGra, 28, 164002

Boyarsky, A., Lesgourgues, J., Ruchayskiy, O., \& Viel, M. 2009a, PhRvL, 102, 201304

Boyarsky, A., Ruchayskiy, O., \& Shaposhnikov, M. 2009b, ARNPS, 59, 191 Boylan-Kolchin, M., Bullock, J. S., \& Kaplinghat, M. 2012, MNRAS, 422, 1203

Bridle, S., Balan, S. T., Bethge, M., et al. 2010, MNRAS, 405, 2044

Brouzakis, N., Tetradis, N., \& Tzavara, E. 2008, JCAP, 4, 8

Bulbul, E., Markevitch, M., Foster, A., et al. 2014, ApJ, 789, 13

Carlesi, E., Knebe, A., Lewis, G. F., Wales, S., \& Yepes, G. 2014a, MNRAS, 439, 2943 
Carlesi, E., Knebe, A., Lewis, G. F., \& Yepes, G. 2014b, MNRAS, 439, 2958

Chang, F., jen Chen, C., \& jen Lu, C. 2004, Computer Vision and Image Understanding, 93, 206

Clarkson, C., Ellis, G. F. R., Faltenbacher, A., et al. 2012, MNRAS, 426, 1121

Dyer, C. C., \& Roeder, R. C. 1972, ApJL, 174, L115

Dyer, C. C., \& Roeder, R. C. 1973, ApJL, 180, L31

Elahi, P. J., Mahdi, H. S., Power, C., \& Lewis, G. F. 2014, MNRAS, 444, 2333

Elahi, P. J., Thacker, R. J., \& Widrow, L. M. 2011, MNRAS, 418, 320

Ellis, R. S. 2010, RSPTA, 368, 967

Finkbeiner, D. P., Goodenough, L., Slatyer, T. R., Vogelsberger, M., \& Weiner, N. 2011, JCAP, 5, 2

Flaugher, B. 2005, IJMPA, 20, 3121

Fleury, P., Dupuy, H., \& Uzan, J.-P. 2013, PhRvD, 87, 123526

Gillis, B., \& Taylor, A. 2016, MNRAS, 456, 2518

Grenon, C., \& Lake, K. 2010, PhRvD, 81, 023501

Gruen, D., Seitz, S., Brimioulle, F., et al. 2014, MNRAS, 442, 1507

Heymans, C., Van Waerbeke, L., Bacon, D., et al. 2006, MNRAS, 368, 1323

Heymans, C., Van Waerbeke, L., Miller, L., et al. 2012, MNRAS, 427, 146

Hildebrandt, H., van Waerbeke, L., \& Erben, T. 2009, A\&A, 507, 683

Ishigaki, M., Kawamata, R., Ouchi, M., et al. 2015, ApJ, 799, 12

Kang, X., Macciò, A. V., \& Dutton, A. A. 2013, ApJ, 767, 22

Kantowski, R. 1998, ApJ, 507, 483

Kantowski, R., \& Thomas, R. C. 2001, ApJ, 561, 491

Kantowski, R., Vaughan, T., \& Branch, D. 1995, ApJ, 447, 35

Klypin, A., Gottlöber, S., Kravtsov, A. V., \& Khokhlov, A. M. 1999, ApJ, 516,530

Lewis, A. 2009, MNRAS, 398, 471

Libeskind, N. I., Di Cintio, A., Knebe, A., et al. 2013, PASA, 30, 39

Lovell, M. R., Eke, V., Frenk, C. S., et al. 2012, MNRAS, 420, 2318

Lovell, M. R., Frenk, C. S., Eke, V. R., et al. 2014, MNRAS, 439, 300

LSST Science Collaboration, Abell, P. A., Allison, J., et al. 2009 , arXiv:0912.0201

Macciò, A. V., Ruchayskiy, O., Boyarsky, A., \& Muñoz-Cuartas, J. C. 2013, MNRAS, 428, 882
Mahdi, H. S., van Beek, M., Elahi, P. J., et al. 2014, MNRAS, 441, 1954

Massey, R., Heymans, C., Bergé, J., et al. 2007, MNRAS, 376, 13

Meneghetti, M., Bartelmann, M., Dahle, H., \& Limousin, M. 2013, SSRv, 177,31

Meures, N., \& Bruni, M. 2012, MNRAS, 419, 1937

Moore, B., Ghigna, S., Governato, F., et al. 1999, ApJL, 524, L19

Nottale, L. 1982a, A\&A, 110, 9

Nottale, L. 1982b, A\&A, 114, 261

Nottale, L. 1983, A\&A, 118, 85

Obreschkow, D., Power, C., Bruderer, M., \& Bonvin, C. 2013, ApJ, 762, 115

Power, C. 2013, PASA, 30, 53

Power, C., Navarro, J. F., Jenkins, A., et al. 2003, MNRAS, 338, 14

Press, W. H., Teukolsky, S. A., Vetterling, W. T., \& Flannery, B. P. 2007 Numerical Recipes: The Art of Scientific Computing (3rd ed.; New York: Cambridge Univ. Press)

Schmidt, F., Leauthaud, A., Massey, R., et al. 2012, ApJL, 744, L22

Schneider, A., Anderhalden, D., Macciò, A. V., \& Diemand, J. 2014, MNRAS, 441, L6

Schneider, A., Smith, R. E., Macciò, A. V., \& Moore, B. 2012, MNRAS, 424, 684

Schneider, P. 2003, arXiv:astro-ph/0306465

Shao, S., Gao, L., Theuns, T., \& Frenk, C. S. 2013, MNRAS, 430, 2346

Sheldon, E. S., Johnston, D. E., Scranton, R., et al. 2009, ApJ, 703, 2217

Springel, V. 2005, MNRAS, 364, 1105

Tomita, K., Asada, H., \& Hamana, T. 1999, PThPS, 133, 155

Umetsu, K., Medezinski, E., Nonino, M., et al. 2014, ApJ, 795, 163

Viel, M., Markovič, K., Baldi, M., \& Weller, J. 2012, MNRAS, 421, 50

Vogelsberger, M., \& Zavala, J. 2013, MNRAS, 430, 1722

Vogelsberger, M., Zavala, J., \& Loeb, A. 2012, MNRAS, 423, 3740

Watanabe, K., \& Tomita, K. 1990, ApJ, 355, 1

Weinberg, S. 1976, ApJL, 208, L1

Xu, D. D., Mao, S., Wang, J., et al. 2009, MNRAS, 398, 1235

Yang, L. F., Neyrinck, M. C., Aragón-Calvo, M. A., Falck, B., \& Silk, J. 2015, MNRAS, 451, 3606 